\title{
Papilomatosis respiratoria recurrente: análisis del impacto económico en el tratamiento quirúrgico
}

Juan Antonio Lugo ${ }^{1}$, Noemí Sainz ${ }^{2}$, Ricardo González Heredia ${ }^{3}$, Melissa Sánchez Guerrero ${ }^{4}$, Víctor Mendoza Ibarra ${ }^{4}$, Alejandra Pineda ${ }^{4}$

\section{RESUMEN}

Objetivo: Evaluar el impacto económico que genera el tratamiento quirúrgico de la papilomatosis respiratoria recurrente en el tercer nivel de atención.

La papilomatosis respiratoria recurrente es una entidad prevalente. El impacto económico que genera se encuentra principalmente en la necesidad de las repetidas intervenciones quirúrgicas que requiere a lo largo de su evolución, además de la morbilidad que ocasiona esta en el paciente.

Materiales y métodos: Este estudio se realizó en el Hospital de Especialidades N ${ }^{\circ} 2$, Centro Médico Nacional del Noroeste, IMSS. Diseño retrospectivo, observacional, trasversal. Muestreo no probabilístico por serie consecutiva de casos. En la descripción general del estudio, se revisaron los expedientes clínicos de los pacientes con diagnóstico de papilomatosis respiratoria recurrente atendidos en el servicio de esta institución de enero de 2010 a enero de 2012. Se tomaron datos como la edad, sexo, manejo quirúrgico y/o terapia coadyuvante, y costo generado por su atención quirúrgica exclusivamente. Resultados: De un total de 103 expedientes revisados, solo 39 se encontraron completos y cumplieron con los criterios de inclusión: 16 (41\%) eran de sexo femenino y $23(59 \%)$, de sexo masculino. Se encontraron 27 casos (69\%) juveniles y $12(30.8 \%)$ adultos. Solo 19 casos (48.7\%) recibieron terapia coadyuvante. En 15 casos (38.5\%) se practicó traqueotomía. El costo directo por intervención quirúrgica de resecciones fue de $\$ 322,943.92$ (22,190 USD) en el grupo juvenil y de $\$ 55,225.33$ (3,790 USD) en el grupo adulto.

Conclusiones: El tratamiento quirúrgico de la papilomatosis respiratoria recurrente representa una carga financiera y una gran morbilidad tanto para las instituciones de salud como para la familia.

Palabras clave: Papilomatosis respiratoria recurrente; tratamiento; costo (Fuente: DeSC BIREME).

\section{Recurrent respiratory papillomatosis: analysis of the economic impact generated by surgical treatment}

\section{ABSTRACT}

Objective: To evaluate the economic impact generated by surgical treatment of recurrent respiratory papillomatosis in the tertiary care level.

Recurrent respiratory papillomatosis is a prevalent entity. The economic impact it generates is mainly due to the need for repeated surgical interventions throughout its progression, in addition to the morbidity it causes to the patient. Materials and methods: This retrospective, observational, cross-sectional study was carried out at the Specialty Hospital No. 2, National Medical Center of the Northwest, Mexican Social Security Institute (IMSS), using a non-probability sampling by consecutive case series. In the general description of the study, clinical records of patients diagnosed with recurrent respiratory papillomatosis treated at this institution from January 2010 to January 2012 were reviewed. Data such as age, gender, surgical management and/or adjuvant therapy, and cost generated by exclusive surgical care, were collected. Results: Out of a total of 103 reviewed files, only 39 were complete and met the inclusion criteria: 16 (41\%) were female and $23(59 \%)$ were male. Twenty-seven (27) cases (69\%) were juvenile patients and $12(30.8 \%)$ were adults. Only 19 cases (48.7\%) received adjuvant therapy. Tracheotomy was performed in 15 cases (38.5\%). The direct cost for resection surgery was $\$ 322,943.92$ (22,190 USD) in the juvenile group and $\$ 55,225.33$ (3,790 USD) in the adult group.

Conclusions: Surgical treatment for recurrent respiratory papillomatosis represents a financial burden and great morbidity for both health institutions and the family.

Keywords: Recurrent respiratory papillomatosis; treatment; cost (Source: MeSH NML).

1. Médico Adscrito y Titular al Servicio de Otorrinolaringología del Hospital de Especialidades $N^{\circ} 2$ "Luis Donaldo Colosio Murrieta”, Unidad Médica de Alta Especialidad, CMNNO. Cd Obregón. Sonora, México.

2. Médico adscrito al Servicio de Otorrinolaringología, Unidad Médica de Alta Especialidad, Hospital de Especialidades $\mathrm{N}^{\circ} 2$ “Luis Donaldo Colosio Murrieta", CMNNO, Cd Obregón. Sonora, México.

3. Médico Especialista en Medicina Familiar, Profesor del Centro de Investigación Educativa Formación Docente, Instituto Mexicano del Seguro Social, Cd Obregón. Sonora, México.

4. Médico Residente del Servicio Otorrinolaringología, Unidad Médica de Alta Especialidad, Hospital de Especialidades $\mathrm{N}^{\circ} 2$ “Luis Donaldo Colosio Murrieta", CMNNO, Cd Obregón. Sonora, México. 


\section{INTRODUCCIÓN}

La papilomatosis respiratoria recurrente (PRR) es una enfermedad que afecta principalmente la laringe y la tráquea y en ocasiones, los bronquios y el parénquima pulmonar. Se caracteriza por la aparición de crecimientos papilomatosos de aspecto similar al de las verrugas. Estos papilomas pueden causar una obstrucción respiratoria potencialmente mortal o/y cambios en la voz ${ }^{(1,2)}$. Es una enfermedad potencialmente devastadora, con una morbilidad significativa. Aunque la papilomatosis respiratoria recurrente se considera una enfermedad benigna, los papilomas se pueden malignizar en un $3 \%$ a un $5 \%$ de los pacientes ${ }^{(3)}$. La papilomatosis respiratoria recurrente tiene una distribución etaria bimodal, al presentarse con frecuencia en niños menores de cinco años o en adultos entre 20 y 30 años Se ha descrito una incidencia de 4,3 por 100000 por año en niños y del 1,8 por 100000 en adultos ${ }^{(4)}$. El agente causal primario es el virus del papiloma humano (HPV), un virus pequeño, sin envoltura, con cápside icosahédrica y ADN circular de doble hélice. El virus invade las células epiteliales y puede existir dentro de su huésped en forma activa o latente. El virus del papiloma humano es el mismo virus asociado con verrugas en la piel, condiloma genital y cáncer cervical.

Aunque hasta el momento se han identificado alrededor de 90 subtipos diferentes del virus del papiloma humano (5). La mayoría de los casos de papilomatosis respiratoria recurrente son dos virus: el HPV-6 y el HPV-11. El tipo 11 aparece como el más virulento de los dos subtipos, asociado con una presentación temprana, una actividad de la enfermedad más prolongada, una mayor cantidad de procedimientos quirúrgicos, una tasa de mortalidad más alta y una mayor frecuencia de malignización ${ }^{(6)}$. En la papilomatosis respiratoria recurrente de aparición en la juventud, la transmisión puede ser secundaria al contacto directo con los papilomas en el canal del parto infectado por VPH cervical materno, sin embargo la cesárea no se ha comprobado que prevenga la aparición (7). En el caso de la papilomatosis respiratoria recurrente de aparición en la adultez, no se establecieron de modo apropiado las formas de transmisión. Los mecanismos postulados incluyen la activación de un virus latente que se encuentra presente desde el nacimiento, o la infección adquirida en la adolescencia o adultez como resultado de un contacto oral o sexual ${ }^{(8)}$. Los síntomas más frecuentes de la papilomatosis respiratoria recurrente incluyen ronquera progresiva, estridor y dificultad respiratoria.

Con menor frecuencia, la papilomatosis respiratoria recurrente se puede presentar con tos crónica, neumonía recurrente, retraso del crecimiento, disnea y disfagia. El diagnóstico se realiza mediante visualización con nasolaringoscopia flexible o laringoscopia directa. La biopsia de las lesiones es útil para la confirmación histológica y para excluir la transformación maligna. Los objetivos del tratamiento son aliviar la obstrucción de las vías respiratorias, mejorar la calidad de voz y facilitar la remisión ${ }^{(9)}$.

El tratamiento generalmente incluye la cirugía citorreductora repetida de los papilomas bajo anestesia general. Los pacientes pediátricos pueden necesitar varios procedimientos durante muchos años. Se han propuesto varios agentes como adyuvantes de la cirugía citorreductora. Como agentes antivirales: ribavirina, aciclovir, cidofovir además otras sustancias como interferón alfa, indol 3-carbinol y terapia fotodinámico. Se han asociado varios efectos secundarios con el uso de estos agentes disponibles además de una mejoría parcial de un alto porcentaje de pacientes. Estos efectos secundarios incluyeron náuseas, vómitos, dolor abdominal, insuficiencia renal aguda, hepatitis y neutropenia ${ }^{(10,11)}$. A pesar de que es una enfermedad benigna que por lo general implica la laringe, el virus del papiloma humano, tiene un curso clínico impredecible, tiende a repetirse y extenderse por todo el tracto respiratorio digestivo y pueden someterse a conversión maligna. Además, se pone un pesada carga emocional a los pacientes y sus familias cuando Se necesitan cirugías repetidas, y su coste económico es alta, estimado en 150 millones dólares anualmente ${ }^{(12)}$. El costo anual de un solo caso de papilomatosis respiratoria recurrente Juvenil es $\$ 57,996$ (rango, $32.407 \$-94.114$ dólares). El costo anual de JORRP en los Estados Unidos es de entre \$ 40 millones y $\$ 123$ millones en función de la prevalencia ${ }^{(13,14)}$. El objetivo de nuestro estudio fue identificar el impacto financiero que genera el tratamiento quirúrgico de la papilomatosis respiratoria recurrente en nuestro hospital.

\section{MATERIALES Y MÉTODOS}

Este estudio se realizó en el servicio de otorrinolaringología del hospital de especialidades $\mathrm{N}^{\circ} 2$, fue un estudio retrospectivo, observacional, trasversal. Con un muestreo no probabilístico por serie consecutiva de casos Se revisaron los expediente clínicos de los pacientes con diagnóstico de papilomatosis respiratoria recurrente atendidos en el servicio de esta institución de enero 2010 a enero 2012. Se incluyeron datos como edad, sexo, manejo quirúrgico y/o terapia coadyuvante y costo generado por su atención quirúrgica exclusivamente, se realizó una análisis estadístico con el SPSS versión 20 , se obtuvieron medidas de tendencia central como medias, modas, promedios y se aplicó chi cuadrado para establecer diferencia significativa entre el grupo pediátrico contra el grupo adulto tomando como diferencia significativa un valor de $\mathrm{p} \leq 0.05$.

\section{RESULTADOS}

De un total de 103 expedientes revisados, solo 39 se encontraron completos y cumplieron los criterios de inclusión, 16 (41\%) sexo femenino y $23(59 \%)$ sexo masculino (Tabla 1). El promedio de edad fue de 24.74 años y por género. De acuerdo a la categoría de inicio de la enfermedad se encontró juvenil 27 (69\%) y adulto 12 (30.8\%) (Figura 1). Respecto al uso de terapia adyuvante en nuestra serie, encontramos que solo 19 casos $(48.7 \%)$ lo recibió, mientras que $20(51.3 \%)$ pacientes solo recibió tratamiento quirúrgico (Tabla 2). De total de 39 pacientes a $15(38.5 \%)$ se practicó traqueotomía, mientras que 
24 casos $(61.5 \%)$ no se realizó En nuestra revisión solo evaluamos el costo directo que implica las cirugías de resecciones, observándose un mayor costo en el grupo juvenil $\$ 322,943.92$ (22,190 USD) comparado con el grupo adulto $\$ 55,225.33(3,790$ USD) (Figura 2$)$.
Al realizar un análisis con Chi 2 referente al costo, se encontró diferencia significativa entre las categorías Juvenil vs Adulto con una P .001 con IC 95\% en relación estrecha con el número de cirugías realizadas.

Tabla 1. Promedio de edad y de género de 39 pacientes con PRR del Servicio de ORL, HE. №2 IMSS. Sonora, México

$\begin{array}{cccccc}\text { Sexo } & \mathbf{N} & \text { Media } & \text { Desv típ } & \text { Mínimo } & \text { Máximo } \\ \text { Masculino } & 23.00 & 27.65 & 20.28 & 4.00 & 68.00 \\ \text { Femenino } & 16.00 & 20.56 & 17.38 & 3.00 & 53.00 \\ \text { Total } & 39.00 & 24.74 & 19.23 & 3.00 & 68.00\end{array}$

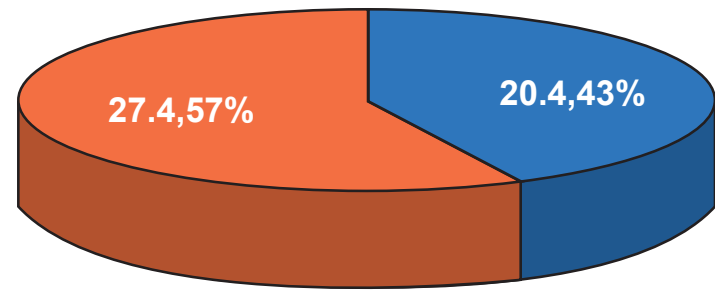

\section{Juvenil}

Adulto

Figura 1. Distribución según de la categoría de la enfermedad de 39 pacientes con PRR del servicio de ORL, H.E. № 2, IMSS. Sonora, México

Tabla 2. Promedio de edad y de género de 39 pacientes con PRR del Servicio de ORL, HE. №2 IMSS. Sonora, México

$\begin{array}{ccc} & \text { Frecuencia } & \text { Porcentaje } \\ \text { Sí } & 19 & 48.7 \% \\ \text { No } & 20 & 51.3 \% \\ \text { Total } & 39 & 100.00\end{array}$

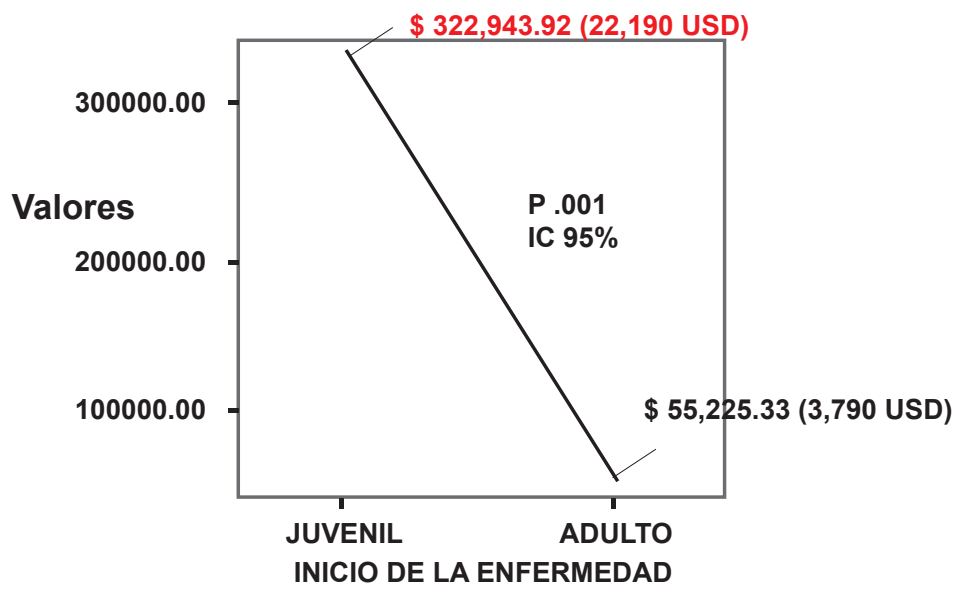

Figura 2. Costo promedio cirugía de resección por tipo de PRR en 39 pacientes del servicio de ORL, H.E. NO 2, IMSS. Sonora, México 


\section{DISCUSIÓN}

La PRR tiene una distribución etaria bimodal, al presentarse con frecuencia en niños menores de cinco años o en adultos entre 20 y 30 años Se ha descrito una incidencia de 4,3 por 100000 por año en niños y del 1,8 por 100000 en adultos.

Nuestro estudio fue similar a lo reportado en la literatura, donde encontramos que del total de 39 pacientes con PRR, hasta un $69.2 \%$ de éstos correspondían a la presentación juvenil y $30.8 \%$ adulto.

La distribución por género en nuestro estudio no mostró diferencia a las publicaciones vistas en la literatura, donde se refiere que hay diferencia entre género en el grupo juvenil; nosotros encontramos 14 femeninos y 13 masculinos, a diferencia en el grupo adulto donde si existe diferencia entre género, encontramos un predominio sexo masculino con 10 y solo 2 del sexo femenino. 4 En la población infantil no parece haber diferencias entre los niños y las niñas pero en adultos hay un predominio masculino 14 .

Las indicaciones ampliamente aceptadas para la aplicación de un tratamiento adyuvante son: necesidad de más de 4 procedimientos quirúrgicos por año, el rápido crecimiento de la papilomatosis con compromiso de la vía aérea o la extensión distal multicéntrico de la enfermedad. En relación a el porcentaje de pacientes que recibieron terapia coadyuvante correspondió a un $48.7 \%$, en total, en su mayoría tipo juvenil; $66 \%$ tipo juvenil y $25 \%$ tipo adulto, considerando únicamente al grupo infantil, nuestro resultados se encuentra muy por arriba de la publicación hecha por Schraff, encontró que el 10\% a 20\% de los pacientes con papilomatosis respiratoria recurrente recibían tratamiento antiviral adyuvante ${ }^{(11)}$. En el Hospital de Pittsburgh desde 1984 a 1994 evaluaron 35 pacientes con PRR de los cuales 13(37\%) recibieron traqueotomía, en nuestros 39 casos, 15 (38.5\%) se practicó traqueotomía En nuestro estudio se encontró un $38.5 \%$ correspondiendo al tipo Juvenil, porcentaje similar a lo reportado por shapiro (16).

El costo del tratamiento de la PRR es elevado, y está supeditado al número de resecciones que se realizan en el paciente, aunado al costo de hospitalizaciones $e$ insumos que estos conlleva, sin incluir las morbilidades y carga familiar que esto implica. En nuestra revisión solo evaluamos el costo directo que implica las cirugías de resecciones, observándose un mayor costo en el grupo juvenil $\$ 322,943.92$ (22,190 USD) comparado con el grupo adulto $\$ 55,225.33(3,790$ USD), relacionado con el número de cirugías. Además, supone un pesada carga emocional a los pacientes y sus familias cuando se necesitan cirugías repetidas, y su coste económico es alta, estimado en 150 millones dólares anualmente ${ }^{(12)}$. El costo anual de un solo caso de papilomatosis respiratopria recurrente Juvenil es $\$$ 57,996 (rango, $32.407 \$-94.114$ dólares). El costo anual de JORRP en los Estados Unidos es de entre $\$ 40$ millones y $\$$ 123 millones en función de la prevalencia ${ }^{(13)}$.
En nuestra revisión solo evaluamos el costo directo que implica las cirugías de resecciones, observándose un mayor costo en el grupo juvenil $\$ 322,943.92$ (22,190 USD) comparado con el grupo adulto $\$ 55,225.33$ (3,790 USD), Al realizar un análisis con Chi 2 referente al costo, se encontró diferencia significativa a favor a las categorías Juvenil vs Adulto con una P .001 con IC 95\% en relación estrecha con el número de cirugías realizadas.

En un estudio publicado por Hu D, se obtuvieron estimaciones de costos para la papilomatosis respiratoria recurrente juvenil, de por vida de $\$ 131.910$ UDS, con un amplio rango de 54.800- 276,170.UDS El costo médico directo de por vida que se asocia en caso nuevos de JORRP durante un período representativo de 1 año es de 82.2 millones de UDS. Cuando se usan valores de límite superior e inferior para la incidencia de JORRP y el costo de por vida por caso, oscila entre $\$ 4.4-\$ 900$ millones USD ${ }^{(17)}$.

En conclusión nuestro estudio, no encontró diferencia significativa, referente al género en la categoría, sin embargo, al separar los grupos, se encontró, que de los 12 casos adultos 10 pertenecían al género masculino; la categoría con mayor frecuente de presentación, es la Juvenil, similar a lo reportado por otros autores.

En nuestro estudio encontramos que los mayores costos se ven en los casos de la PRR juvenil, igual a lo encontrado por otros autores, ello debido a que se necesita una frecuencia mayor de intervenciones quirúrgicas. La papilomatosis respiratoria recurrente, es una enfermedad con un gran impacto económico y familiar, ya que por tratarse de una enfermedad crónica, que hasta hoy en día no existe una cura, su tratamiento se encuentra dirigido solo a manejo paliativo, y evitar mayores complicaciones, implicando un problema con gran carga familiar y costo en los sistemas de salud.

\section{REFERENCIAS BIBLIOGRÁFICAS}

1. Hadha NK, James AL. Tratamiento antiviral adyuvante para la papilomatosis respiratoria recurrente . En: La Biblioteca Cochrane Plus, 2008 Número 2. Oxford: Update Software Ltd. Disponible en:http://www.update-software.com.

2. Derkay CS, Malis DJ, Zalzal G, Wiatrak BJ, Kashima HK, Coltrera MD.A staging system for assessing severity of disease and response to therapyin recurrent respiratory papillomatosis. Laryngoscope 1998;108(6):935-7.

3. Kimberlin DW. Current status of antiviral therapy for juvenileonset recurrent respiratory papillomatosis. Antiviral Research 2004;63(3):141-51.

4. Shykhon $M$, Kuo $M$, Pearman K. Recurrent respiratory papillomatosis. Clinical Otolaryngology and Allied Sciences. 2002;27:237-43.

5. Menzo S, Monachetti A, Trozzi C, Ciavattini A, Carloni G, Varaldo PE, et al. Identification of six putative novel human papillomaviruses (HPV) and characterization of candidate HPV type 87. Journal of Virology 2001;75(23):119139.,

6. Rabah R, Lancaster W, Thomas R, Gregoire L. Human papillomavirus-11-associated recurrent respiratory papillomatosis is more aggressive than human papillomavirus6-associated disease. Pediatric and Developmental Pathology. 2001;4(1):68-72. 
7. Silverberg $M$, Thorsen $P$, Lindeberg $H$, Grant L, Shah $K$ Condyloma in pregnancy is strongly predictive of juvenileonset recurrent respiratory papillomatosis. Obstetrics and Gynecol 2003;101(4):645-52.

8. Kashima H, Shah F, Lyles A, Glackin R, Muhammad N, Turner L, et al. A comparison of risk factors in juvenile-onset and adultonset recurrent respiratory papillomatosis. Laryngoscope. 1992;102(1):9-13.

9. Derkay C. Recurrent respiratory papillomatosis. Laryngoscope. 2001;111:57-69

10. Mehta D. British National Formulary. 46th Edition. British Medical Association and Royal Pharmaceutical Society of Great Britain, 2003.

11. Schraff S, Derkay S, Burke B, Lawson L. American Society of Pediatric Otolaryngology members' experience with recurrent respiratory papillomatosis and the use of adjuvant therapy. Arch Otolaryngol Head Neck Surg. 2004;130(9):1039-42.

12. Derkay C, Wiatrak B. Recurrent Respiratory Papillomatosis: A Review, Laryngoscope, 2008;118:1236 -47

13. Bishai D, Kashima H, Shah K. The cost of juvenile-onset recurrent respiratory papillomatosis. Arch Otolaryngol Head Neck Surg. 2009;135(2):208.

14. Derkay C. Task force on recurrent respiratory papillomas. A preliminary report. Arch Otolaryngol Head Neck Surg. 1995;121(12):1386-91.

15. Graupp M, Gugatschka M, Kiesler K, Reckenzaun E, Hammer G, Friedrich $G$. Experience of 11 years use of cidofovir in recurrent respiratory papillomatosis. Eur Arch Otorhinolaryngol. 2013;270(2):641-6.

16. Shapiro A. Tracheotomy in children with juvenile-onset recurrent respiratory papillomatosis: the Children's Hospital of Pittsburgh experience. Ann Otol Rhinol Laryngol. 1996;105(1):1-5
17. Hu D, Goldie S. The economic burden of noncervical human papillomavirus disease in the United States. Am J Obstet Gynecol. 2008;198(5):.e1-e7.

\section{Fuentes de Financiamiento}

Este artículo ha sido financiado por los autores.

\section{Conflicto de interés}

Los autores declaran no tener ningún conflicto de interés.

\section{Correspondencia:}

Juan Antonio Lugo Machado

Dirección: Prolongación Hidalgo Bellavista -Cajeme 85130

Ciudad Obregón, Sonora.

Teléfono: +6441559891

Correo electrónico: otorrinox@gmail.com

\section{Recibido: 06 de julio de 2017 Evaluado: 08 de julio de 2017 Aprobado: 15 de setiembre de 2017}

(c) La revista. Publicado por Universidad de San Martín de Porres, Perú. (cc) вr Licencia de Creative Commons Artículo en acceso abierto bajo términos de Licencia Creative Commons Atribución 4.0 Internacional. (http://creativecommons.org/licenses/by/4.0/) 